ARTICLE

https://doi.org/10.1038/s41467-022-28392-8

\title{
Anionic character of the conduction band of sodium chloride
}

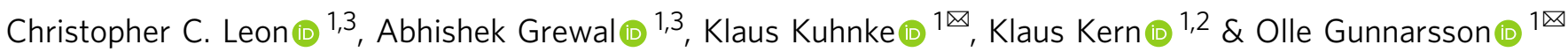

The alkali halides are ionic compounds. Each alkali atom donates an electron to a halogen atom, leading to ions with full shells. The valence band is mainly located on halogen atoms, while, in a traditional picture, the conduction band is mainly located on alkali atoms. Scanning tunnelling microscopy of $\mathrm{NaCl}$ at $4 \mathrm{~K}$ actually shows that the conduction band is located on $\mathrm{Cl}^{-}$because the strong Madelung potential reverses the order of the $\mathrm{Na}^{+} 3 \mathrm{~s}$ and $\mathrm{Cl}^{-}$4s levels. We verify this reversal is true for both atomically thin and bulk $\mathrm{NaCl}$, and discuss implications for II-VI and I-VII compounds.

\footnotetext{
${ }^{1}$ Max-Planck-Institut für Festkörperforschung, Heisenbergstraße 1, 70569 Stuttgart, Germany. ${ }^{2}$ Institut de Physique, École Polytechnique Fédérale de Lausanne, 1015 Lausanne, Switzerland. ${ }^{3}$ These authors contributed equally: Christopher C. Leon, Abhishek Grewal. ${ }^{凶}$ email: k.kuhnke@fkf.mpg.de; 
nsulating compounds host a rich and very varied physics. One distinguishes between ionic ${ }^{1}$, Slater ${ }^{2}, \operatorname{Mott}^{3}$ and charge transfer $^{4-7}$ insulators, all involving quite different mechanisms and having different properties. Slater and Mott insulators primarily involve a partly filled band, in which antiferromagnetic or Coulomb interactions open up a gap. The late transition metal oxides are examples of charge transfer insulators, involving the $\mathrm{O} 2 p$ band and the, partly filled, transition metal $3 d$ band. Coulomb interactions are essential also in this case. Ionic insulators, e.g., alkali halides, appear to be conceptually simpler. In a one-particle picture, all bands are completely full or empty, and Coulomb interactions are less essential. There is a large charge transfer from the alkali atoms to the halogen atoms, which become positively and negatively charged ions. The valence band is primarily of halogen $p$ character. This band is separated from the conduction band by a large gap. The conduction band is assumed to have mainly alkali character, involving an empty alkali $s$ level outside a full shell. While this picture has been presented in many text books and publications ${ }^{1,8-13}$ this view has occasionally been questioned on theoretical grounds. Slater and Shockley ${ }^{14}$ suggested a different picture for $\mathrm{NaCl}$, in which the conduction band also has a substantial $\mathrm{Cl} 4 s$ character. Clark ${ }^{15,16}$, de Boer and de Groot $^{17,18}$, as well as Olsson et al. ${ }^{19}$. performed band structure calculations for $\mathrm{NaCl}$ and concluded that the conduction band is actually mainly located on the $\mathrm{Cl}^{-}$ions. These theoretical results, however, seem to have been largely overlooked or ignored, possibly because of problems with uniquely assigning charges to ions.

We present a heuristic calculation which suggests that a very large Madelung potential ${ }^{20}(\approx 9 \mathrm{eV})$ can reverse the order of the $\mathrm{Cl}^{-} 4 s$ (above the vacuum level) and $\mathrm{Na}^{+} 3 s($ at $\approx-5 \mathrm{eV}$ ) levels and imply a conduction band of mainly $\mathrm{Cl}$ character. (The Madelung potential is the potential at any ion position in an ionic crystal due to the combined electrostatic potentials of the infinite number of ions in the crystal.) However, such a calculation (and similar considerations) alone is not decisive proof of this reality because of confounding factors such as the large spatial extent of $s$ orbitals and nonunique assignments of charges to ions. For exactly this reason, we perform an experimental study of the conduction band of $\mathrm{NaCl}$ using scanning tunnelling microscopy (STM) providing a real space picture of states, which are centred on the $\mathrm{Cl}^{-}$ions across the entire band gap.

\section{Results}

Despite the many STM studies addressing the structure and growth of $\mathrm{NaCl}$ films ${ }^{21-25}$, such a detailed study of the $\mathrm{NaCl}$ conduction band has not yet been performed, possibly because it is very challenging to obtain atomic resolution on $\mathrm{NaCl}$ at positive bias (e.g., $U>1 \mathrm{~V})^{22}$. These difficulties preclude a straightforward spatial mapping of the $\mathrm{NaCl}$ conduction band by STM and an identification of the relative local density of states at $\mathrm{Na}$ and $\mathrm{Cl}$ positions. The instability of the tunnelling condition necessary to achieve atomic resolution simply prohibits addressing the conduction band directly.

We circumvent this problem by making the bias very positive to approach the conduction band very closely without exceeding the band gap, and harnessing our understanding of the tunnelling process under these conditions. We present STM images of $\mathrm{NaCl}(100)$ on $\mathrm{Au}(111)$, varying the bias over a large range. They show that tunnelling happens through the $\mathrm{Cl}^{-}$ions, even for energies just below the conduction band, indicating that the conduction band also has mainly $\mathrm{Cl}$ character.

To support this conclusion we present tight-binding (TB) calculations for two models of a $\mathrm{NaCl}$ film on a Au surface, with the conduction band mainly on either the $\mathrm{Na}^{+}$or $\mathrm{Cl}^{-}$ions. These calculations show that features in STM images for energies just below the conduction band indeed are located on the same ions as the conduction band itself.
We compare $\mathrm{NaCl}$ on $\mathrm{Au}$ and bulk $\mathrm{NaCl}$ theoretically. We find that the order of the $\mathrm{Na} 3 s$ and the $\mathrm{Cl} 4 s$ levels are reversed by similar amounts in both cases, as discussed extensively in Supplementary Note 2. The conclusions obtained for $\mathrm{NaCl}$ on $\mathrm{Au}$ should therefore also apply to bulk $\mathrm{NaCl}$.

We furthermore extend the discussion of $\mathrm{NaCl}$ to II-VI and other I-VII compounds, suggesting that the conduction band could have a substantial weight on the anion also in these cases.

STM topography of $\mathrm{NaCl}$ over the entire band gap. Because few experimental techniques can characterise the conduction band character of the alkali halides with atomic resolution, we investigated the $\mathrm{NaCl}(100)$ surface with STM. Since directly accessing the conduction band of $\mathrm{NaCl}(100)$ involves high electric fields that destroy its structural integrity ${ }^{22}$, we do measurements at voltages as close as possible to the conduction band edge while staying within the band gap of $\mathrm{NaCl}$. Topographs are measured at constant current whose colour scale represent tip-sample distance. By repeating the measurements at certain voltages, the tip quality was monitored and care was taken that the relatively high bias voltages did not modify the tip character or shift the tip apex. Similarly, the tip drift over time, typical for STM, was monitored and corrected in the presented data.

Figure la shows a region of $\mathrm{Au}(111)$ covered with a 2 monolayer (ML) thick (apparent height: $309 \pm 3 \mathrm{pm}$ ) $\mathrm{NaCl}$ terrace, consistent with prior studies ${ }^{26}$. Atop the $\mathrm{NaCl}$ terrace, the protrusions form a square lattice whose unit cell length is $4 \AA$ (Fig. 1a). The square lattice is reproduced in reciprocal space (Fourier transform inset, Fig. 1a).

We survey the electronic structure of the system at selected positions marked by grey and black rings in Fig. 1a. The pronounced surface state of $\mathrm{Au}(111)$ shows an onset at $-500 \mathrm{mV}$ measured at the grey ring. This onset shifts to $\approx-250 \mathrm{mV}$ when measured on $\mathrm{Au}-\mathrm{NaCl}$ at the black ring. This shift originates from the Pauli interaction experienced between $\mathrm{NaCl}$ and $\mathrm{Au}(111)$. $\mathrm{NaCl}$ acts as a dielectric, polarises and weakens the image potential arising from $\mathrm{Au}(111)$ alone $\mathrm{e}^{27,28}$ (inset Fig. 1b) measured at the black ring. A large range voltage scan of differential conductance indicating density of states of $\mathrm{NaCl}$ is shown in Fig. $1 \mathrm{~b}$. Arrows point to the valence and conduction band onsets at $-3.0 \mathrm{~V}$ and $+3.6 \mathrm{~V}$.

The region marked by the white box, in the fcc region of herringbone reconstruction of $\mathrm{Au}(111)$, in Fig. 1a is scanned with high stability and signal to noise ratio (Fig. 1c-h) for applied sample voltages from $-3 \mathrm{~V}$ to $+2.5 \mathrm{~V}$, respectively. At applied bias of $-3.0 \mathrm{~V}$ (Fig. 1c), a voltage close to the valence band edge of $\mathrm{NaCl}$ (Fig. 1b), a square lattice of protrusions is seen whose intensity is proportional to the $z$-corrugation. Each protrusion is assigned to $\mathrm{Cl}$. The assignment of protrusions at negative voltage to $\mathrm{Cl}$ is in agreement with many earlier studies ${ }^{19,22,29}$. We do not observe any change in position of $z$-corrugation at negative voltages (Fig. 1c-e). These measurements further corroborate the valence band being mainly $\mathrm{Cl}$ in character.

We now consider the same region scanned at positive bias. Simple but ultimately misleading electrostatic arguments suggest that the topographic protrusions would become centred over $\mathrm{Na}^{+}$ ions. For instance, electron extraction at negative bias is helped at the $\mathrm{Cl}^{-}$positions with the $\mathrm{Cl}^{-}$being relatively electron-rich. Electron injection at positive bias is helped at the $\mathrm{Na}^{+}$positions with the $\mathrm{Na}^{+}$being relatively electron-poor. Thus, one might anticipate that inverting the applied bias polarity should result in an inversion of the topographic contrast due to $z$-corrugation resulting from $\mathrm{Na}^{+}$ions instead of $\mathrm{Cl}^{-}$ions.

We perform this experiment and obtain evidence to the contrary. Fig. 1f-h show the result of probing the $\mathrm{NaCl}$ layer at positive bias up to $+2.5 \mathrm{~V}$. By comparing these topographic 

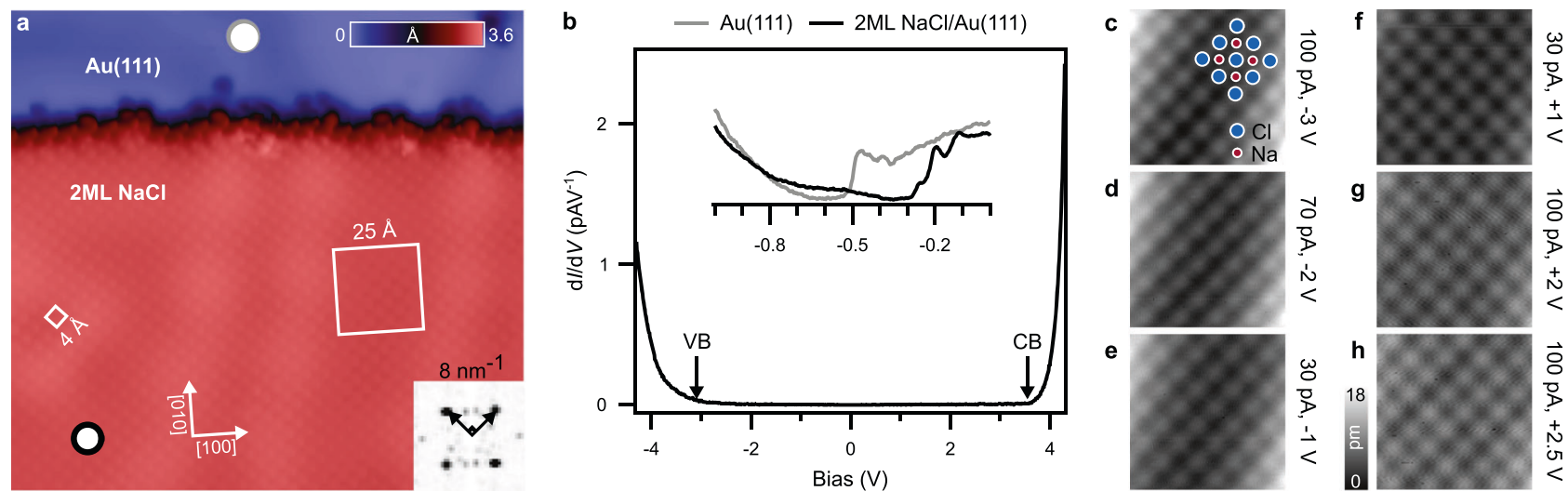

Fig. 1 STM of $2 \mathbf{M L} \mathbf{~ N a C l ( 1 0 0 )}$ on $\mathbf{A u ( 1 1 1 )}$ with topography and $\mathbf{d} \mathbf{I} / \mathbf{d} \mathbf{V}$ measurements. a Overview of the measurement area $\left(150 \times 140 \AA^{2}, I_{T}=8 \mathrm{pA}\right.$ $V_{S}=-50 \mathrm{mV}$ ). $25 \times 25 \AA^{2}$ region marked, investigated for bias values between $-3.0 \mathrm{~V}$ and $+2.5 \mathrm{~V}$. Inset: Fourier transform of $\mathbf{f}$ centred over a

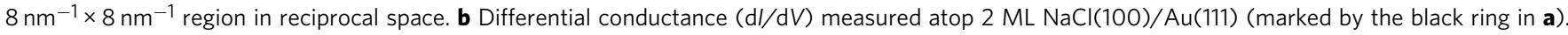
The arrows point to onset of valence band (VB) and conduction band (CB). Inset. Surface state features of Au(111) and related interface state of $2 \mathrm{ML}$ $\mathrm{NaCl}(100) / \mathrm{Au}(111)$ measured at the positions marked in $\mathbf{a}$ by a grey ring and a black ring, respectively. Vertical scale: arbitrary units. c-h Raw data showing grayscale topography images obtained for sample bias between $-3.0 \mathrm{~V}$ and $+2.5 \mathrm{~V}$ as indicated. The red and blue dots in $\mathbf{c}$ mark the positions of $\mathrm{Na}$ and $\mathrm{Cl}$ ions of the $\mathrm{NaCl}(100)$ lattice, respectively. The corresponding tunnelling parameters (current, voltage) are indicated next to each topography image.
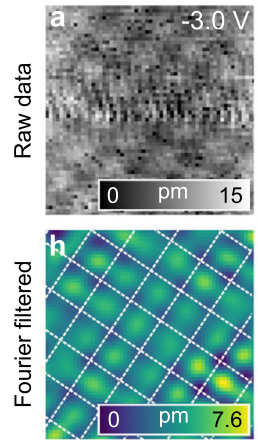
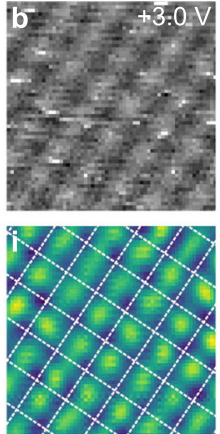
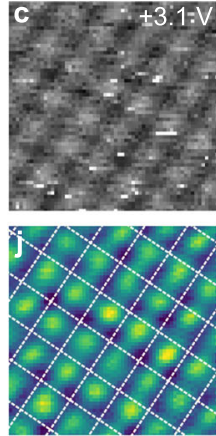
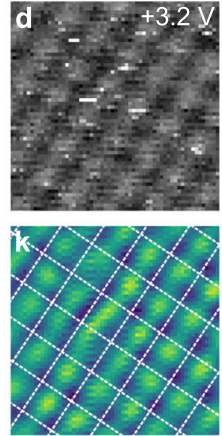
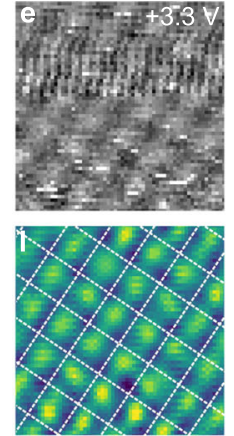
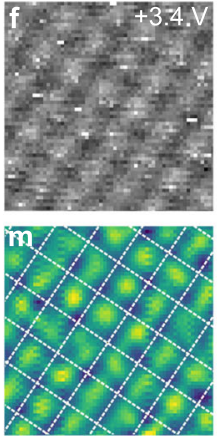
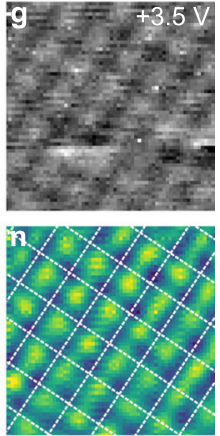

Fig. 2 STM of $3 \mathbf{~ M L ~ N a C l ( 1 0 0 )}$ on Au(111) at voltages close to both edges of the band gap. a-g Raw (grayscale) and $\mathbf{h}-\mathbf{n}$ Fourier filtered (coloured) at the indicated voltages, $I_{T}=15 \mathrm{pA}$. Scale: $24 \times 24 \AA^{2}$. The white dashed lines mark the position of $\mathrm{Na}$ ion rows of the $\mathrm{NaCl}(100)$ lattice.

measurements with those obtained at negative bias (Fig. 1c-e), it is evident that the protrusions remain centred over $\mathrm{Cl}$ and do not shift over to $\mathrm{Na}$. If the conduction band were cationic in character, one would have expected to see topographic intensity over the $\mathrm{Na}^{+}$ions. Yet no contrast inversion is observed when the bias polarity is inverted. In the band gap of $\mathrm{NaCl}$, the $z$ corrugation is correlated to the electronic states of energetically nearest neighbouring bands. Thus, the experiment shows that the electronic states of $\mathrm{NaCl}$ have substantial weight on $\mathrm{Cl}$ rather than $\mathrm{Na}$, irrespective of bias polarity. When one tunnels at energies within the band gap of $\mathrm{NaCl}$, electron transport in $\mathrm{NaCl}$ is dominated by the role of $\mathrm{Cl}^{-}$ions at both negative and positive bias. This implies that both the valence and conduction bands of $\mathrm{NaCl}$ are anionic in character, a point that is to be emphasised.

The expected role of $\mathrm{Na}^{+}$ions in the conduction band of $\mathrm{NaCl}$ asserted by many textbooks and publications is simply absent. Rather, we see evidence for $\mathrm{Cl}$ functioning as both an electron donor and acceptor in $\mathrm{NaCl}$. We conceptualise the electronic structure of $\mathrm{NaCl}$ as that of spectator $\mathrm{Na}^{+}$cations holding together $\mathrm{Cl}^{-}$anions that are mainly responsible for electron conduction.

To further evidence the markedly anionic (rather than cationic) character of the $\mathrm{NaCl}$ conduction band, we expand the voltage range of the measurements to $+3.5 \mathrm{~V}$ in exchange for greatly reduced tip stability and signal to noise ratio. However, we still keep the absolute piezo drift $\left(0.014 \mathrm{~nm} \mathrm{~min}^{-1}\right)$ during data acquisition to be significantly smaller than the $\mathrm{Cl}^{-}-\mathrm{Na}^{+}$ion spacing $(\approx 0.3 \mathrm{~nm})$ and along a direction that avoids introducing spurious contrast inversions due to drift. These efforts are shown in Fig. 2 in which a different sample area of $3 \mathrm{ML} \mathrm{NaCl}(100)$ $(483.5 \pm 5.4 \mathrm{pm}$ thickness) on $\mathrm{Au}(111)$ is scanned with a newly prepared tip at select voltages and analysed with Fourier filtered scans. As discussed by Lauwaet et al. ${ }^{26}$, the interface state (see inset Fig. 1b) wave function for $\mathrm{NaCl}(100)$ on $\mathrm{Au}(111)$ does not extend further than two $\mathrm{NaCl}$ layers. In the discussion in Supplementary Note 2, we conclude that electronically, $2 \mathrm{ML}$ and $3 \mathrm{ML} \mathrm{NaCl}$ are identical. In contrast to Fig. 1, the scans in Fig. 2 are taken across the well-defined fcc and hcp regions of the $\mathrm{Au}(111)$ herringbone reconstruction.

At bias $>+2 \mathrm{~V}$, the risk of tip changes and spontaneous defect creation in the $\mathrm{NaCl}$ layer increase considerably. As seen in Fig. 2a-g, while tip transients do prevent smooth scanning at high bias, they do not completely destroy the important atomic resolution. The low and high frequencies are Fourier filtered to mitigate noise and to clearly highlight the periodic $z$-corrugation. The measurements in Fig. 2 attain atomic resolution on $\mathrm{NaCl}$ even at higher bias than the ones in Fig. 1 . Once again, at $-3 \mathrm{~V}, z$ corrugation is assigned to $\mathrm{Cl}$ positions and does not shift to $\mathrm{Na}$ positions (lattice positions marked by white dashed linse in Fig. $2 \mathrm{~h}-\mathrm{n}$ ) at any of the indicated applied voltages in Fig. 2. Repeating these measurements at other voltages between $-3.0 \mathrm{~V}$ to $+3.5 \mathrm{~V}$ reveals no evidence for electron density maxima at $\mathrm{Na}$. The results in Fig. 2 show that electron density remains centred 


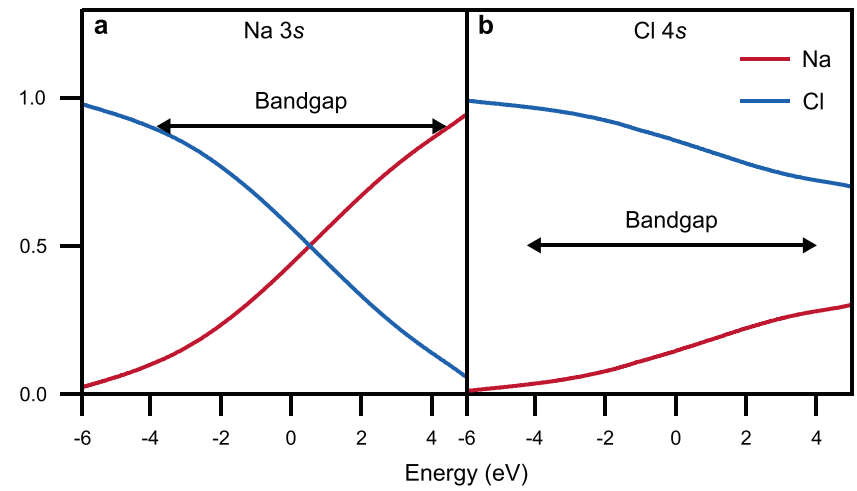

Fig. 3 Character of the gap states of $\mathrm{NaCl}$ including Madelung potential. Normalised relative weight of gap states on $\mathrm{Na}$ and $\mathrm{Cl}$ atoms in the outermost $\mathrm{NaCl}$ layer as a function of energy, $E$, in or close to the gap (indicated by the arrows). The parameters were adjusted so that the conduction states have mainly $\mathbf{a} \mathrm{Na}$ character and $\mathbf{b} \mathrm{Cl}$ character. The Figure illustrates that electrons travelling through the gap at energies close to the conduction band, have similar character as those in the conduction band.

over $\mathrm{Cl}$, even up to $+3.5 \mathrm{~V}$ (Fig. $2 \mathrm{~g}, \mathrm{n}$ ) which is quite close to the empirically measured conduction band edge $(\approx+3.6 \mathrm{~V})$ shown in Fig. 1b. The second data set thus demonstrates that the conclusions about tunnelling being weighted on $\mathrm{Cl}$ in $\mathrm{NaCl}$ holds more generally and is observed on different $\mathrm{NaCl}$ layer heights, on fcc as well as hcp areas and by using a tip after different preparation.

Tight binding calculations. We now perform tight-binding calculations for a model of a $3 \mathrm{ML} \mathrm{NaCl}(100)$ film on a $\mathrm{Au}(111)$ surface (see Supplementary Note 1). We use two different sets of parameters for the $\mathrm{NaCl}$ film, resulting in a conduction band mainly of either $\mathrm{Na}$ $3 s$ or $\mathrm{Cl} 4 s$ character. In the first set we use $3 s$ and $3 p$ basis states on the $\mathrm{Cl}$ atoms, and in the second set the $\mathrm{Cl} 3 s$ basis states are replaced by $4 s$ basis states. States in the energy gap of $\mathrm{NaCl}$ are described as linear combinations of valence and conduction states.

In Fig. 3a, we use conventional parameters, including $3 s$ and $3 p$ states on the $\mathrm{Cl}$ atoms ${ }^{12}$ and neglect the Madelung potential. (Note, that in the calculation with $\mathrm{Cl} 3 s$ and $3 p$ orbitals only, the conduction band is repelled upwards by these states. Including higher states on $\mathrm{Cl}$ would tend to have the opposite effect, requiring a higher $\mathrm{Na} 3 s$ level to obtain the correct band gap. This may then require a contribution from the Madelung potential also in this case). As we increase the energy through the gap, the character of the state changes from mainly $\mathrm{Cl}$ character close to the valence band to mainly $\mathrm{Na}$ character close to the conduction band, in contradiction to the experimental finding.

Figure $3 \mathrm{~b}$ shows results for the second set of parameters, including $3 p$ and $4 s$ states on the $\mathrm{Cl}$ atoms. In this case, it is essential to include a Madelung potential to obtain a conduction band of mainly $\mathrm{Cl}$ character and a correct band gap. The result is that the gap states have mainly $\mathrm{Cl}$ character throughout the whole gap, in agreement with experiment. This then provides further evidence that not only the gap states but also the conduction band has mainly $\mathrm{Cl}$ character. We emphasise again, the inclusion of $\mathrm{Cl}$ $4 s$ states in these calculations.

Tunnelling through an insulator band gap proceeds via the electronic states of the energetically nearest neighbouring bands. If $\mathrm{NaCl}$ were composed of a $\mathrm{Cl}^{-}$based valence band and a $\mathrm{Na}^{+}$based conduction band, an atomically resolved STM map of it would show voltage-dependent contrast that inverts at a specific voltage in the band gap suggested in Fig. 3a. However, this inversion does not happen in the experiment. In fact, the same type of ion appears bright at energies near both the valence and conduction bands, corroborating the picture that both bands have most of their weight on the same type of ion, specifically $\mathrm{Cl}^{-}$in the case of $\mathrm{NaCl}$ (Fig. 3b).

\section{Discussion}

Naively applying classical electrostatics, one would predict that the negatively charged electrons avoid the negatively charged $\mathrm{Cl}^{-}$ in favour of the positively charged $\mathrm{Na}^{+}$. Moreover, because $\mathrm{Cl}^{-}$ has a full shell electron configuration $[\mathrm{Ne}] 3 s^{2} 3 p^{6}$, the extra tunnelling electron must be accommodated in the higher energy $\mathrm{Cl}$ $4 s$ orbital, which also appears to impose an insurmountable energetic cost.

To understand why the $\mathrm{Cl} 4 s$ states might, nevertheless, be so important, we make a few very simple considerations. For fully ionised atoms (in the sense of $\mathrm{Cl}^{-}$and $\mathrm{Na}^{+}$having exact full shell electronic configurations), the Madelung potential is $8.9 \mathrm{eV}$, leading to a strong upward shift of the $\mathrm{Na} 3 \mathrm{~s}$ level, which for a neutral atom is just at $-5.1 \mathrm{eV}^{30}$. Even if the $3 s$ orbital relaxes somewhat, it may then be pushed above the vacuum level by the Madelung potential.

Meanwhile, the $4 s$ level of a free $\mathrm{Cl}^{-}$ion is above the vacuum level. The issue is whether the Madelung potential can pull this level below the $\mathrm{Na} 3 s$ level. To puzzle this out, we use a thought experiment to increase the nuclear charge of $\mathrm{Cl}$ by two. This turns $\mathrm{Cl}^{-}$into $\mathrm{K}^{+}$, for which the $4 s$ level is at $-4.1 \mathrm{eV}^{30}$, below the (raised) $\mathrm{Na} 3 \mathrm{~s}$ level. The question is if the Madelung potential has a similar attractive effect on the $\mathrm{Cl} 4 s$ level. We use Slater's rules ${ }^{31}$ to approximate the $4 s$ state of $\mathrm{K}^{+}$as $\phi_{4 s} \sim r^{2.7} \exp (-2.2 r / 3.7)$, where 2.2 is the effective nuclear charge and $n=3.7$ is the effective principal quantum number. We then calculate the attractive potential on the $4 s$ state due to increasing the nuclear charge of $\mathrm{Cl}$ by $\Delta Z=2$. The magnitude of this potential is indeed comparable to the Madelung potential of $8.9 \mathrm{eV}$. In reality, the atoms are not fully ionised and so the Madelung potential is smaller. Simple estimates suggest, however, that this effect and several other smaller effects do not change the picture in an essential way (see Supplementary Note 3 ). The K 4 s orbital is very extended and complicates the interpretation of these results. Nevertheless, these simple considerations make it plausible that the Madelung potential can reverse the order of the $\mathrm{Cl}^{-} 4 s$ level and the $\mathrm{Na}^{+} 3 s$ level, resulting in a conduction band of mainly $\mathrm{Cl}$ character in $\mathrm{NaCl}$. This is confirmed by the STM measurements in Figs. 1 and 2 that show well-defined features centred on the $\mathrm{Cl}$ ions. This interpretation is robust because the STM measurements are performed in real space and do not depend on interpretations expressed in terms of orbitals.

We now discuss differences between bulk $\mathrm{NaCl}$ and $\mathrm{NaCl}$ on $\mathrm{Au}$, using tight-binding calculations described in the Supplementary Note 2. For a $\mathrm{NaCl}$ film on $\mathrm{Au}$, we find an appreciable charge transfer to the Au substrate, consistent with an observed substantial reduction of the work function. This raises the potential of the outer layers of the film substantially, but the effect on the potential difference between the $\mathrm{Na}$ and $\mathrm{Cl}$ sites in the outermost layer is very small. The assumed smaller lattice parameter of the $\mathrm{NaCl}$ film on $\mathrm{Au}$ increases the Madelung potential, while the finite thickness of the film reduces it. Adding up all effects, we find that the tendency to put the conduction band on the $\mathrm{Cl}$ atoms should be comparable (difference on the order of $0.1 \mathrm{eV}$, see Supplementary Table IV) for bulk $\mathrm{NaCl}$ and for $\mathrm{NaCl}$ on $\mathrm{Au}$, implying that the effects observed here should apply also to bulk $\mathrm{NaCl}$.

In nanoscience, a $\mathrm{NaCl}$ film is often used as a buffer between a substrate and a molecule to be studied ${ }^{32,33}$. Due to the large $\mathrm{NaCl}$ gap, the applied voltage is typically such that the electrons 
tunnel through the $\mathrm{NaCl}$ gap. The way the substrate couples to the molecule then depends on whether the corresponding electrons mainly tunnel through the $\mathrm{Cl}^{-}$or $\mathrm{Na}^{+}$ions. The results above should then be important for interpreting such experiments.

In contrast to the discussion above, in nanoscience it is often assumed that the electrons tunnel through a band gap via some state, not well specified, being different from both the valence and conduction states ${ }^{34}$. To address this issue, we could have included additional basis states, which would then have to be orthogonal to the valence and conduction states of the $\mathrm{NaCl}$ film. However, such states would necessarily be higher in energy than the conduction states already included. This implies that the additional basis states would not be very important for describing gap states, which are located below the conduction band. By contrast, the explicit use of just valence and conduction states as basis states should already give a good qualitative picture of what happens when tunnelling through the gap, and such calculations are relatively easy to interpret.

In view of these results for $\mathrm{Na}^{+} \mathrm{Cl}^{-}$it is interesting to discuss $\mathrm{Mg}^{2+} \mathrm{O}^{2-}$. In analogy to the discussion above, we do the thought experiment of increasing the nuclear charge of $\mathrm{O}^{2-}$ by three, obtaining $\mathrm{Na}^{+}$with its $3 s$ level at $-5.1 \mathrm{eV}$. The attractive interaction of this additional nuclear charge with the $3 s$ level is $20 \mathrm{eV}$, comparable to the Madelung potential $23.9 \mathrm{eV}$ for the ions $\mathrm{Mg}^{2+}$ and $\mathrm{O}^{2-}$. This suggests that the conduction band of $\mathrm{MgO}$ could be located on oxygen, involving mainly $\mathrm{O} 3 s$ levels. Indeed, this is what was found in band structure calculations by de Boer and de Groot $^{35}$. It would then be very interesting to check experimentally the character of the conduction bands both for the II-VI and for other I-VII ionic compounds.

Beyond these simple salts, it would indeed be fruitful to further experiment with materials whose valence and conduction bands take on unusual combinations of cationic and anionic character, e.g., that of the valence band being cationic ${ }^{36}$, or that of the conduction band being anionic ${ }^{37}$. There may also be complementary mechanisms to probe that induce electronic structure rearrangements like that of the Madelung potential.

To summarise, using STM we have shown that the conduction band of $\mathrm{NaCl}$ has mainly $\mathrm{Cl}$ character, contrary to widespread belief. That the conduction band can be anionic in character is related to the large Madelung potential, which pushes the $\mathrm{Na} 3 \mathrm{~s}$ level upwards and pulls the $\mathrm{Cl} 4 s$ level downwards, which for a free $\mathrm{Cl}^{-}$species is above the vacuum level. We provided a back of the envelope calculation to make it plausible that the Madelung potential could actually reverse the order of the $\mathrm{Cl}^{-} 4 s$ and the $\mathrm{Na}^{+} 3$ s levels, leading to this result. This electronic structure may couple into other physical processes. Incidentally, it is the negative ion vacancies that are important to electrical conduction near the melting point of some alkali halides ${ }^{38}$.

\section{Methods}

Sample preparation and details of STM measurements. The experiments were carried out with a home-built low-temperature STM operated at $T=4.3 \mathrm{~K}$ in ultrahigh vacuum $\left.\left(<10^{-11}\right) \mathrm{mbar}\right)^{39}$. The $\mathrm{Au}(111)$ single-crystal $(>99.999 \%)$ sample was cleaned by repeated cycles of $\mathrm{Ar}^{+}$ion sputtering at $10^{-6}$ mbar range argon pressure with $600 \mathrm{eV}$ acceleration energy and subsequent annealing to $873 \mathrm{~K}$. The sample heating and cooling rate was about $1 \mathrm{~K} / \mathrm{s}$. $\mathrm{NaCl}$ was evaporated thermally from a Knudsen cell held at $900 \mathrm{~K}$, with the $\mathrm{Au}(111)$ surface held at $300 \mathrm{~K}$, to obtain defect-free, (100)-terminated $\mathrm{NaCl}$ islands. An electrochemically etched gold wire $^{40}$ ( $99.95 \%$ purity) was used as tip in the experiment. To ensure a metallic tip, the Au wire is further prepared by controlled tip indentations $(1-3 \mathrm{~nm}$, $V=50-100 \mathrm{mV})$ in $\mathrm{Au}(111)$ until atomic resolution is obtained at tunnelling current set point: $I_{T}=10 \mathrm{pA},+1 \mathrm{~V}$. The text always specifies bias voltages of the metal substrate with respect to the grounded tip. Differential conductance $(\mathrm{d} I / \mathrm{d} V)$ spectra were measured using a standard lock-in technique with a bias modulation of $V_{\text {rms }}=4 \mathrm{mV}$ (Fig. 1b inset) and $V_{\text {rms }}=10 \mathrm{mV}$ (Fig. 1b) at $629 \mathrm{~Hz}$. Scanning tunnelling microscopy/spectroscopy data were analysed using self-written MATLAB code.

\section{Data availability}

The data generated in this study have been deposited in the figshare database under accession code https://doi.org/10.6084/m9.figshare.17159228 ${ }^{41}$.

Received: 15 November 2021; Accepted: 19 January 2022; Published online: 21 February 2022

\section{References}

1. Seitz, F. The Modern Theory Of Solids (McGraw-Hill Book Co., 1940).

2. Slater, J. C. Magnetic effects and the hartree-fock equation. Phys. Rev. 82, 538-541 (1951).

3. Mott, S. N. F. Metal-insulator Transitions (Taylor \& Francis, 1974).

4. Fujimori, A., Minami, F. \& Sugano, S. Multielectron satellites and spin polarization in photoemission from Ni compounds. Phys. Rev. B 29 5225-5227 (1984).

5. Fujimori, A. \& Minami, F. Valence-band photoemission and optical absorption in nickel compounds. Phys. Rev. B 30, 957-971 (1984).

6. Sawatzky, G. A. \& Allen, J. W. Magnitude and origin of the band gap in $\mathrm{NiO}$ Phys. Rev. Lett. 53, 2339-2342 (1984).

7. Zaanen, J., Sawatzky, G. A. \& Allen, J. W. Band gaps and electronic structure of transition-metal compounds. Phys. Rev. Lett. 55, 418-421 (1985).

8. Harrison, W. A. Solid State Theory (McGraw-Hill, 1970).

9. Stoneham, A. M. Theory of Defects in Solids: Electronic Structure of Defects in Insulators and Semiconductors. Theory of Defects in Solids (Oxford University Press, 2001)

10. Ching, W. Y., Gan, F. \& Huang, M.-Z. Band theory of linear and nonlinear susceptibilities of some binary ionic insulators. Phys. Rev. B 52, 1596-1611 (1995).

11. Elliott, S. The Physics And Chemistry Of Solids (Wiley, 1998).

12. Harrison, W. Elementary Electronic Structure https://doi.org/10.1142/4121 (World scientific, 1999).

13. Grosso, G. \& Parravicini, G. P. Solid State Physics (Elsevier Science, 2000).

14. Slater, J. C. \& Shockley, W. Optical absorption by the alkali halides. Phys. Rev. 50, 705-719 (1936).

15. Clark, T. A. Calculation Of The Electronic Energy Band Structure Of Sodium Chloride (Iowa State University, 1968).

16. Clark, T. D. \& Kliewer, K. L. Augmented plane wave calculation of the electronic energy bands of NaCl. Phys. Lett. A 27, 167-168 (1968).

17. de Boer, P. K. \& de Groot, R. A. The origin of the conduction band in table salt. Am. J. Phys. 67, 443-445 (1999).

18. de Boer, P. K. \& de Groot, R. A. With a grain of salt. Phys. Lett. A 256, 227-229 (1999).

19. Olsson, F. E., Persson, M., Repp, J. \& Meyer, G. Scanning tunneling microscopy and spectroscopy of $\mathrm{NaCl}$ overlayers on the stepped $\mathrm{Cu}(311)$ surface: experimental and theoretical study. Phys. Rev. B 71, 075419 (2005).

20. Madelung, E. Das elektrische Feld in Systemen von regelmäßig angeordneten Punktladungen. Phys. Z. 19, 524 (1918).

21. Glöckler, K., Sokolowski, M., Soukopp, A. \& Umbach, E. Initial growth of insulating overlayers of $\mathrm{NaCl}$ on $\mathrm{Ge}(100)$ observed by scanning tunneling microscopy with atomic resolution. Phys. Rev. B 54, 7705-7708 (1996).

22. Hebenstreit, W. et al. Atomic resolution by STM on ultra-thin films of alkali halides: experiment and local density calculations. Surf. Sci. 424, 321-328 (1999).

23. Bennewitz, R. et al. Atomically resolved edges and kinks of $\mathrm{NaCl}$ islands on $\mathrm{Cu}(111)$ : Experiment and theory. Phys. Rev. B 62, 2074-2084 (2000).

24. Repp, J. Rastertunnelmikroskopie und -spektroskopie an Adsorbaten auf Metall- und Isolatoroberflächen (Freie Universität Berlin, 2002).

25. Lauwaet, $\mathrm{K}$. et al. Resolving all atoms of an alkali halide via nanomodulation of the thin $\mathrm{NaCl}$ film surface using the $\mathrm{Au}(111)$ reconstruction. Phys. Rev. B 85, 245440 (2012)

26. Lauwaet, K., Schouteden, K., Janssens, E., Haesendonck, C. V. \& Lievens, P. Dependence of the $\mathrm{NaCl} / \mathrm{Au}(111)$ interface state on the thickness of the $\mathrm{NaCl}$ layer. J. Phys. Condens. Matter 24, 475507 (2012).

27. Diekhöner, L. et al. Surface states of cobalt nanoislands on $\mathrm{Cu}(111)$. Phys. Rev. Lett. 90, 236801 (2003).

28. Repp, J., Meyer, G. \& Rieder, K.-H. Snell's law for surface electrons: refraction of an electron gas imaged in real space. Phys. Rev. Lett. 92, 036803 (2004).

29. Olsson, F. E. \& Persson, M. A density functional study of adsorption of sodium-chloride overlayers on a stepped and a flat copper surface. Surf. Sci. 540, 172-184 (2003).

30. Moore, C. E. Ionization Potentials and Ionization Limits Derived from the Analyses of Optical Spectra. (National Bureau of Standards, 1970).

31. Slater, J. C. Atomic shielding constants. Phys. Rev. 36, 57-64 (1930).

32. Repp, J., Meyer, G., Stojković, S. M., Gourdon, A. \& Joachim, C. Molecules on insulating films: scanning-tunneling microscopy imaging of individual molecular orbitals. Phys. Rev. Lett. 94, 026803 (2005). 
33. Liljeroth, P., Repp, J. \& Meyer, G. Current-induced hydrogen tautomerization and conductance switching of naphthalocyanine molecules. Science 317, 1203-1206 (2007).

34. Chen, G. et al. Spin-triplet-mediated up-conversion and crossover behavior in single-molecule electroluminescence. Phys. Rev. Lett. 122, 177401 (2019).

35. de Boer, P. K. \& de Groot, R. A. The conduction bands of $\mathrm{MgO}, \mathrm{MgS}$ and $\mathrm{HfO}_{2}$. J. Phys. Condens. Matter 10, 10241-10248 (1998).

36. Blasse, G., Dirksen, G. J. \& de Korte, P. H. M. Materials with cationic valence and conduction bands for photoelectrolysis of water. Mater. Res. Bull. 16, 991-998 (1981).

37. Zhuravlev, Yu. N. \& Korabel'nikov, D. V. Nature of electronic states and optical functions of sodium oxyanionic compounds. Phys. Solid State 51, 69-77 (2009).

38. Rao, K. V. Electrical conductivity and dielectric properties of $\mathrm{NaCl}, \mathrm{KCl}$, and $\mathrm{KBr}$ single crystals near their melting temperatures. Phys. Status Solidi (a) 30, 391-395 (1975).

39. Kuhnke, K. et al. Versatile optical access to the tunnel gap in a low-temperature scanning tunneling microscope. Rev. Sci. Instrum. 81, 113102 (2010).

40. Yang, B., Kazuma, E., Yokota, Y. \& Kim, Y. Fabrication of sharp gold tips by three-electrode electrochemical etching with high controllability and reproducibility. J. Phys. Chem. C 122, 16950-16955 (2018).

41. Leon, C. C., Grewal, A., Kuhnke, K., Kern, K. \& Gunnarsson, O. Source data for the article: anionic character of the conduction band of sodium chloride. figshare https://doi.org/10.6084/m9.figshare.17159228 (2021).

\section{Author contributions}

A.G. and C.C.L. performed the experimental work and analysed the experimental data. The tight binding calculations were planned and carried out by O.G. C.C.L. and O.G. wrote the manuscript with input from all authors. O.G., K.Ku. and K.Ke. conceived and designed the research programme.

\section{Funding}

Open Access funding enabled and organized by Projekt DEAL.

\section{Competing interests}

The authors declare no competing interests.

\section{Additional information}

Supplementary information The online version contains supplementary material available at https://doi.org/10.1038/s41467-022-28392-8.

Correspondence and requests for materials should be addressed to Klaus Kuhnke or Olle Gunnarsson.

Peer review information Nature Communications thanks the anonymous reviewer(s) for their contribution to the peer review of this work.

Reprints and permission information is available at http://www.nature.com/reprints

Publisher's note Springer Nature remains neutral with regard to jurisdictional claims in published maps and institutional affiliations.

(c) (i)

Open Access This article is licensed under a Creative Commons Attribution 4.0 International License, which permits use, sharing, adaptation, distribution and reproduction in any medium or format, as long as you give appropriate credit to the original author(s) and the source, provide a link to the Creative Commons license, and indicate if changes were made. The images or other third party material in this article are included in the article's Creative Commons license, unless indicated otherwise in a credit line to the material. If material is not included in the article's Creative Commons license and your intended use is not permitted by statutory regulation or exceeds the permitted use, you will need to obtain permission directly from the copyright holder. To view a copy of this license, visit http://creativecommons.org/ licenses/by/4.0/.

(c) The Author(s) 2022 